\title{
Canted Persistent Spin Texture and Quantum Spin Hall Effect in WTe 2
}

\author{
Jose H. Garcia $\odot,{ }^{1}$ Marc Vila $\odot,{ }^{1,2}$ Chuang-Han Hsu, ${ }^{3}$ Xavier Waintal $\odot,{ }^{4}$ Vitor M. Pereira $\odot,{ }^{5,6}$ and Stephan Roche $\odot^{1,7}$ \\ ${ }^{1}$ Catalan Institute of Nanoscience and Nanotechnology (ICN2), CSIC and BIST, Campus UAB, Bellaterra, 08193 Barcelona, Spain \\ ${ }^{2}$ Department of Physics, Universitat Autònoma de Barcelona, Campus UAB, Bellaterra, 08193 Barcelona, Spain \\ ${ }^{3}$ Department of Electrical and Computer Engineering, National University of Singapore, Singapore 117576, Singapore \\ ${ }^{4}$ Université Grenoble Alpes, CEA, IRIG-PHELIQS, 38000 Grenoble, France \\ ${ }^{5}$ Centre for Advanced 2D Materials and Graphene Research Centre, National University of Singapore, Singapore 117546 \\ ${ }^{6}$ Department of Materials Science and Engineering, National University of Singapore, Singapore 117575 \\ ${ }^{7}$ ICREA-Institució Catalana de Recerca i Estudis Avançats, 08010 Barcelona, Spain
}

(Received 2 July 2020; revised 2 October 2020; accepted 12 November 2020; published 18 December 2020)

\begin{abstract}
We report an unconventional quantum spin Hall phase in the monolayer $\mathrm{WTe}_{2}$, which exhibits hitherto unknown features in other topological materials. The low symmetry of the structure induces a canted spin texture in the $y z$ plane, which dictates the spin polarization of topologically protected boundary states. Additionally, the spin Hall conductivity gets quantized $\left(2 e^{2} / h\right)$ with a spin quantization axis parallel to the canting direction. These findings are based on large-scale quantum simulations of the spin Hall conductivity tensor and nonlocal resistances in multiprobe geometries using a realistic tight-binding model elaborated from first-principle methods. The observation of this canted quantum spin Hall effect, related to the formation of topological edge states with nontrivial spin polarization, demands for specific experimental design and suggests interesting alternatives for manipulating spin information in topological materials.
\end{abstract}

DOI: 10.1103/PhysRevLett.125.256603

Introduction.-The prediction of the quantum spin Hall (QSH) insulator state [1-5] and its connection with topological states in strong spin-orbit coupling materials $[6,7]$ sparked an exciting playground for fundamental studies [8-10]. The subsequent demonstration of the existence of topological insulators [6,11-15] then opened a myriad of technological possibilities, since topologically protected states are predicted to carry spin information over unprecedented distances due to a strong resilience to disorder, as long as time-reversal symmetry is preserved $[4,7,16,17]$. But, to date, a QSH effect at room temperature has not yet been experimentally achieved [8,18-26]. Transition metal dichalcogenides (TMDs) in their $1 T^{\prime}$ $\left(P 2_{1} / m\right)$ and $1 T_{d}\left(P m n 2_{1}\right)$ structural phases are currently of much interest due to their unusual spin-charge interconversion characteristics [27-31] and tunable topological phases [28,32-35]. Moreover, recent signatures of the QSH effect up to $\sim 100 \mathrm{~K}$ in monolayer $\mathrm{WTe}_{2}$ [24] promote them as outstanding candidates for realizing a resilient QSH regime. However, the lack of a robust spin Hall conductance quantization insensitive to the device characteristicshallmark of topological physics in the quantum Hall regime [36] - demands in-depth scrutiny of the fundamentals of spin transport in both the topologically trivial and nontrivial regimes, as well as an assessment of possible fundamental limitations [21,37-41].

On the other hand, the traditional spin Hall effect, driven by spin-dependent impurity scattering, is usually associated with spin polarization pointing perpendicular to the conducting plane [9,42]. Some models of 2D QSH systems, such as the Kane-Mele-Haldane Hamiltonian in the absence of Rashba spin-orbit coupling (SOC), are characterized by helical edge states whose spins are also perpendicularly polarized $[1,3]$. However, an out-of-plane spin polarization is not an inherent property of the intrinsic spin Hall effect (quantized or not), but rather a consequence of the underlying symmetries of the crystal. As a matter of fact, different experimental groups recently measured spin Hall conductivities associated with both in- and out-ofplane spin polarizations in few-layer $1 T_{d}$ and $1 T^{\prime}-\mathrm{MoTe}_{2}$, both of similar magnitude, which illustrates the peculiar aspects of bulk spin transport in these materials [27,35,43-45]. To date, little is known about the imprint of the inherently low symmetry of this class of TMDs in the QSH regime. Correlations and substrate effects were found to induce localization of edge modes [38], and resilient in-plane spin states [46], but the impact of low symmetries and the possibility of multiple spin Hall components in the QSH remains to be determined.

In this Letter, we show that the low-symmetry phase $\left(1 T_{d}\right)$ of the $\mathrm{WTe}_{2}$ monolayer leads to an unconventional QSH effect, in which the topological edges states exhibit a canted spin polarization in the $y z$ plane. This differs from the conventional $z$-polarized feature frequently discussed for canonical models of QSH systems. Moreover, the spin Hall conductivity becomes quantized in contrast with other QSH topological insulators [2]. These results emerge from complementary calculations of the spin Hall conductivity 
tensor combined with simulations of nonlocal transport in realistic multiprobe geometries, with and without disorder. The calculations hinge upon an effective four-band tightbinding model that, beyond symmetry, reproduces the essential features of the low-energy band and spin structures of this material. The results further reveal that the spintronic potential of $\mathrm{WTe}_{2}$ is unique even when doped away from the QSH insulator regime, as it displays a persistent spin texture, defined as a constant spin polarization throughout the entire Fermi contour $[7,47,48]$. In addition to numerical calculations with millions of orbitals, we provide analytical connections between the canted spin quantization axis of the topological states and the spin texture of the bulk bands induced by the SOC parameters.

Model and methodology.-We derived a generic densityfunctional theory (DFT)-based four-band tight-binding Hamiltonian on a rectangular lattice, which is applicable to $1 T^{\prime}$ and $1 T_{d}$ TMDs [49]. The spinful model describes the two lowest energy bands belonging to the irreducible representations $A_{g}$ (conduction, mostly of metal $d$ orbital content) and $B_{u}$ (valence, of $p$ orbital content) of the point group $C_{2 h}$. This pair of bands is "inverted" in $\mathrm{WTe}_{2}$ at the $\Gamma$ point, rendering the ground state a QSH topological insulator. The model is similar to symmetry-based models used in recent work $[38,50,51]$. When expanded near the $\Gamma$ point, the Hamiltonian $\mathcal{H}=\mathcal{H}_{0}+\mathcal{H}_{\text {soc }}$ has the following $\boldsymbol{k} \cdot \boldsymbol{p}$ representation:

$\mathcal{H}_{0} \simeq\left(k_{x}^{2}+k_{y}^{2}\right)\left(m_{p} \tau_{0}+m_{d} \tau_{z}\right)+\beta k_{y} \tau_{y}+\delta \tau_{z}+\eta \tau_{x}$,

with $\tau_{i}(i=x, y, z)$ the $2 \times 2$ Pauli matrices in orbital space. The parameters $m_{p}=-0.1050 \mathrm{eV}$ and $m_{d}=-0.5449 \mathrm{eV}$ are related to the effective masses of the valence and conduction bands, $\delta=0.4248 \mathrm{eV}$ describes the degree of band inversion at $\Gamma, \beta=0.4494 \mathrm{eV}$ models the $x-y$ crystalline anisotropy $(\hat{\boldsymbol{x}} \| \boldsymbol{a}$ crystal axis or zigzag direction), and $\eta$ breaks inversion symmetry to describe either the $1 T^{\prime}$ $(\eta=0)$ or $1 T_{d}(\eta=0.0017 \mathrm{eV})$ phase. At the $\boldsymbol{k} \cdot \boldsymbol{p}$ level, the $\mathrm{SOC}$ is given by

$$
\mathcal{H}_{\mathrm{soc}} \simeq\left(\Lambda_{x} k_{y} \sigma_{x}+\Lambda_{y} k_{x} \sigma_{y}+\Lambda_{z} k_{x} \sigma_{z}\right) \otimes \tau_{x},
$$

where $\left(\Lambda_{x}, \Lambda_{y}, \Lambda_{z}\right)=(0.0591,0.0777,-0.1159) \mathrm{eV}$ and the Pauli matrices $\sigma_{i}(i=x, y, z)$ are defined in the spin space. The parameters were determined by fitting the band structure and spin textures to reproduce DFT calculations as described in the Supplemental Material [49].

Figure 1(b) shows a close-up of the model-generated band structure near the Fermi level. The underlying DFT calculation is based on the PBE + HSE functional [52], which places the Fermi level $\left(E_{F}\right)$ near the bottom of the conduction band. The effective model describes accurately the conduction band and energy gap. Each band features two charge pockets symmetrically located away from $\Gamma$, with minima $\left(\varepsilon_{0} \approx-27 \mathrm{meV}\right)$ at the point labeled $Q$ and its (a)
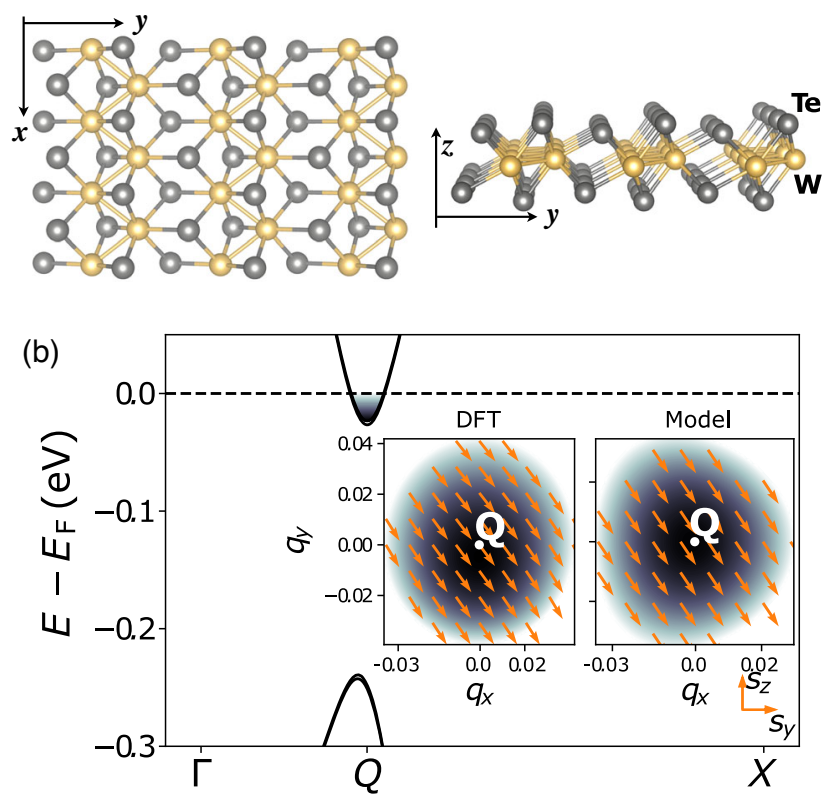

FIG. 1. (a) Scheme of the crystal structure of $\mathrm{WTe}_{2}$ in the $1 T_{d}$ phase. (b) Band structure of $\mathrm{WTe}_{2}$ around the charge pockets formed by the band inversion at $\Gamma$. The conduction band minimum is at the $k$ point $\boldsymbol{Q}=(0.266,0.0)$ with energy $\varepsilon_{0} \approx-27 \mathrm{meV}$. The inset compares the spin textures computed from DFT and the effective model; the color represents the energy with respect to the Fermi level and the arrows the spin orientation in the $y z$ plane (spin projection along $x$ is negligible). The white dots mark the position of the $Q$ point which we have centered at $\boldsymbol{q}=0$. All $k$ points are in units of $\pi / a_{x}$ with $a_{x}$ the lattice constant along the zigzag direction.

time-reversal counterpart (not shown). Though present, the spin-orbit band splitting is small and barely discernible at the scale shown. In the inset, we compare the spin textures at $E_{F}$ for one of the $Q$-centered electron pockets obtained by DFT with that arising from the model. The spin orientations in the $y z$ plane are represented by the orange arrows (despite not strictly zero, the $x$ component is omitted for clarity, as it was found comparatively much smaller in magnitude). In addition to the obvious agreement, it is noteworthy that the spin texture is uniform to a very good approximation. $\mathrm{WTe}_{2}$ is hence a case with a naturally present persistent spin texture which is invariant upon changing $E_{F}$ within the range of energies shown (i.e., low electronic densities). The spins cant at an angle $\theta \approx-56^{\circ}$ with respect to $y$.

The canted spin texture contrasts with the out-of-plane spin polarization in two-dimensional centrosymmetric systems, and also with the in-plane spin-momentum locking in systems with broken inversion symmetry [53], but follows from straightforward symmetry considerations [49]. In systems with multiple vertical mirror symmetries the coefficient $\Lambda_{z}$ of Eq. (2) vanishes, thereby reducing Eq. (2) to a Rasha-like spin-orbit interaction with in-plane spin-momentum locking. Additionally, all the nonzero elements of the spin Hall conductivity tensor describe 
spins pointing out of the plane. Such symmetry constraints are absent in $\mathrm{WTe}_{2}$ monolayers where only a mirror-point symmetry $M_{x}$ remains, allowing for multiple components of the spin texture and different spin polarizations in the spin Hall conductivity tensor.

We next explore the nature of spin transport as $E_{F}$ is varied across the band gap by computing the spin Hall conductivity tensor $\left(\sigma_{i j}^{\alpha}, \alpha=x, y, z\right)$ using the Kubo-Bastin formula implemented for the tight-binding model $[54,55]$ :

$\sigma_{i j}^{\alpha}=-2 \hbar \Omega \int_{-\infty}^{E_{\mathrm{F}}} d E \operatorname{Im}\left(\operatorname{Tr}\left[\delta(E-\mathcal{H}) J_{s, i}^{\alpha} \frac{d G^{+}}{d E} J_{j}\right]\right)$,

where $\Omega$ is the area; $J_{s, i}^{\alpha} \equiv\left\{J_{i}, \sigma_{\alpha}\right\} / 2$ is the $i$ th component of the spin current density operator, with $\alpha=x$, $y, z$ denoting the spin polarization direction; $J_{j} \equiv$ $(i e / \Omega \hbar)\left[\mathcal{H}, R_{j}\right]$ is the $j$ th component of the current density operator, with $e$ the electron charge and $R_{j}$ the position operator [56]. The spectral operators $\delta(E-\mathcal{H})$ and $G^{+} \equiv$ $1 /\left(E-\mathcal{H}+i 0^{+}\right)$are the Dirac delta and the retarded Green's function, respectively. We numerically computed the Kubo-Bastin formula using the kernel polynomial method [53,55-57] with 2000 Chebyshev expansion moments (equivalent to a $5 \mathrm{meV}$ of broadening), on a system containing $4 \times 1000 \times 1000$ orbitals. In addition, we simulated multiterminal nonlocal transport within the Landauer-Büttiker framework as implemented in the Kwant package [58,59], using the six-terminal device geometry shown in the inset of Fig. 3.

Spin Hall conductivity.-Figure 2 shows the nonzero components of the transverse spin Hall conductivity tensor, $\sigma_{x y}^{\alpha}, \alpha \in\{y, z\}$, as $E_{F}$ is varied near and within the band gap. Although both $\sigma_{x y}^{z}$ and $\sigma_{x y}^{y}$ display a plateau in the gap

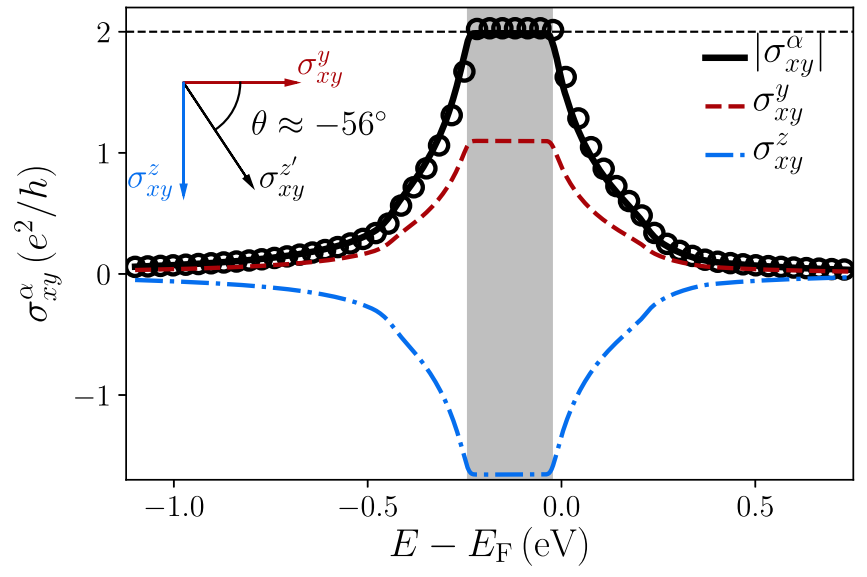

FIG. 2. Spin Hall conductivities $\sigma_{x y}^{y}$ and $\sigma_{x y}^{z}$. The solid line shows the norm of $\left|\sigma_{x y}^{\alpha}\right| \equiv \sqrt{\left(\sigma_{x y}^{y}\right)^{2}+\left(\sigma_{x y}^{z}\right)^{2}}$, the gray area highlights the band gap; the open black circles correspond to $\sigma_{x y}^{z^{\prime}}$. Inset: orientation of the spin of the helical edge states. The calculations were done considering a broadening of $5 \mathrm{meV}$ on a system with $1000 \times 1000 \times 4$ orbitals. region, their values are $-1.65 e^{2} / h$ and $1.1 e^{2} / h$, respectively. This is intriguing since usually, within a topological gap, quantized spin Hall conductivities are integer multiple of the conductance quantum, reflecting the existence of a definite (integer) number of helical edge channels [49].

However, we note that, by definition, each component $\alpha$ of $\sigma_{x y}^{\alpha}$ provides only a measure of the projection of the spin onto the Cartesian direction $\alpha$, because $\sigma_{i j}^{\alpha} \propto J_{s, i}^{\alpha} / J_{j}$ where $J_{s}^{\alpha}$ is the spin current density carrying spins polarized parallel to $\alpha$ in response to a driving charge current $\boldsymbol{J}$. But the choice of Cartesian directions is arbitrary-in fact, the results in Fig. 2 show that a Cartesian system fixed by the orthorhombic axes of the crystal obscures the adequate spin quantization axis in this problem. This is readily confirmed by the fact that, in the gap, $\left|\sigma_{x y}^{\alpha}\right| \equiv \sqrt{\left(\sigma_{x y}^{y}\right)^{2}+\left(\sigma_{x y}^{z}\right)^{2}}$ is indeed quantized at $2 e^{2} / h$ (Fig. 2, solid curve), where the factor of 2 reflects the existence of two counterpropagating modes per edge.

This shows that the interdependence among the magnitudes of the spin Hall conductivity components seen in Fig. 2 has a fundamental origin, namely the presence of spin-canted topological edge states which sustain a $\mathrm{QSH}$ effect in $\mathrm{WTe}_{2}$. From the values of each plateau, we determine that the spin quantization axis is canted at $\arctan \left(\sigma_{x y}^{z} / \sigma_{x y}^{y}\right)=-56^{\circ}$ with respect to the $y$ axis. Notably, this angle matches perfectly with the orientation of the

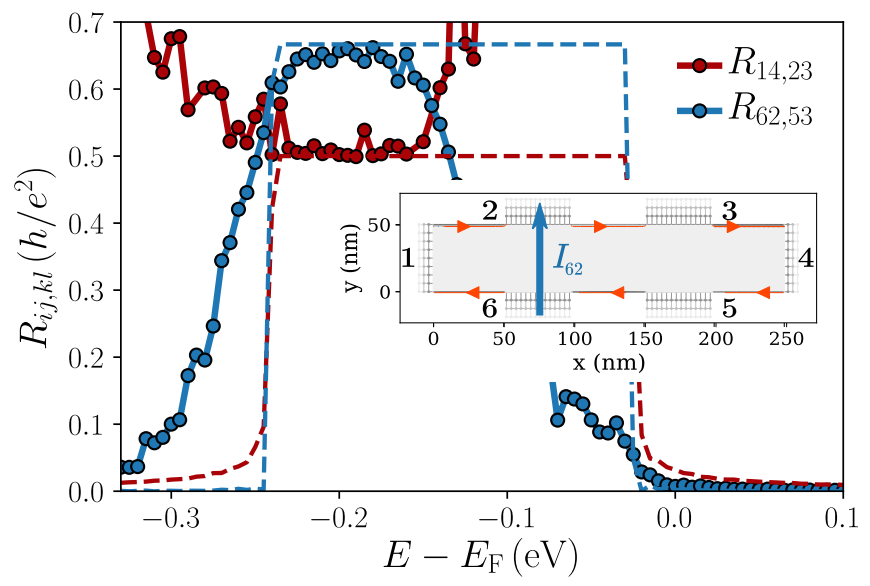

FIG. 3. Nonlocal resistances $R_{i j, k l}=\left(V_{k}-V_{l}\right) / I_{i j}$ calculated in the six-terminal Hall-bar device shown in the inset. The two plateau values $2 h / 3 e^{2}$ and $h / 2 e^{2}$ seen here unequivocally attribute the nonlocal signal to QSH edge states [60]. Solid (dashed) lines correspond to simulations with (without) Anderson disorder (with strength $U=2 \mathrm{eV}$ ). In the inset, the solid (lattice) regions delineate the device (leads). The device is defined on a rectangular lattice (parameters $a_{x}=3.4607 \AA$ and $a_{y}=6.3066 \AA$ ). The device width, interlead separations, and lead widths are all $50 \mathrm{~nm}$. The small horizontal arrows along the top and bottom edges mark the direction of the local, bondprojected spin current density $\boldsymbol{J}_{s}^{z^{\prime}}$ arising as the response to driving charge current from lead 6 to lead 2 . 
persistent spin texture near the bottom of the conduction band, shown earlier in the inset of Fig. 1.

The existence of a quantized plateau at $2 e^{2} / h$ is an indication of spin conservation $[4,61,62]$. To demonstrate this more explicitly, we unitarily transform the Hamiltonian $\mathcal{H}$ with a rotation in spin space about $\hat{x}$, which is effected by the matrix $U(\theta) \equiv \cos [(2 \theta-\pi) / 4] \sigma_{0}-$ $i \sin [(2 \theta-\pi) / 4] \sigma_{x}$, where $\theta \equiv \arctan \left(\Lambda_{z} / \Lambda_{y}\right) \approx-56^{\circ}$ is an angle defined by the SOC parameters in Eq. (2). While $\mathcal{H}_{0}$ is invariant, the SOC term transforms into

$$
\mathcal{H}_{\mathrm{SOC}}^{\prime} \equiv U^{\dagger}(\theta) \mathcal{H} U(\theta)=\Lambda_{x} k_{y} \sigma_{x}+\Lambda_{r} k_{x} \sigma_{z^{\prime}} \tau_{x},
$$

with $\Lambda_{r} \equiv \sqrt{\Lambda_{z}^{2}+\Lambda_{y}^{2}}$ and $\sigma_{z^{\prime}} \equiv U^{\dagger}(\theta) \sigma_{z} U(\theta)$. We now note that $\Lambda_{x}$ is numerically smaller than $\Lambda_{r}$ in $\mathrm{WTe}_{2}$ and, more importantly, $\left|k_{y}\right| \ll\left|k_{x}\right|$ near the bottom of the $Q$ centered electronic pockets. The combined effect is that, over the range of energies shown in Fig. 1, the first term in Eq. (4) is 2 orders of magnitude smaller than the second and thus negligible in practice. Consequently, $\left[\mathcal{H}^{\prime}, \sigma_{z^{\prime}}\right] \approx 0$ so that spin is preserved along the canted $z^{\prime}$ direction to a very good approximation, which has two physical consequences: (i) when $E_{F}$ lies in the conduction band, the carriers have a persistent spin texture directed along $z^{\prime}$ over the entire Fermi contour; (ii) the canting angle is preserved in the QSH regime (when $E_{F}$ lies in the gap), which supports the quantization of the spin Hall conductivity and defines a canted QSH effect. For completeness and further corroboration, we include in Fig. 2 (open circles) the spin Hall conductivity $\sigma_{x y}^{z^{\prime}}$ computed explicitly with the rotated Hamiltonian in Eq. (4). An extended discussion of the relation between spin conservation and the QSH effect is given in Ref. [49].

Chiral transport of spin at the edges.-The topological nature of the electronic states can be unequivocally confirmed by probing nonlocal resistances $R_{\mathrm{nl}}$ in a Hall-bar geometry under different bias conditions: If the nonlocal signal is due only to helical edge states, $R_{\mathrm{nl}}$ should display plateau values uniquely determined by the specific combination of contacts chosen for current injection and nonlocal voltage detection [60]. We employed our effective tightbinding model to compute the nonlocal resistance using the device geometry illustrated in Fig. 3 (inset). To obtain $R_{\mathrm{nl}}$, we first calculate the Landauer-Büttiker transmission probabilities between each pair of leads and build the conductance matrix $G_{i j}[59,63]$ that satisfies the linear system of equations $I_{i}=\sum_{j} G_{i j} V_{j}$, where $I_{i}$ and $V_{j}$ describe the current and voltage at each lead. We then require the current to flow from lead $i$ to $j$ by setting $I_{i}=-I_{j}$ and $I_{k}=0, k \neq i, j$, and calculate the resulting voltages $V_{j}$. The nonlocal resistances are defined as $R_{i j, k l} \equiv\left(V_{k}-V_{l}\right) / I_{i j}$, i.e., current flows from lead $i$ to $j$ and voltage is measured between leads $k$ and $l$. Furthermore, to test the robustness of the nonlocal signal, we included (nonmagnetic) Anderson disorder in the tightbinding Hamiltonian, diagonal in both orbital and spin spaces, whereby a uniformly distributed random energy $U_{r}$ is added at each lattice site, with $U_{r} \in[-U / 2, U / 2]$.

The results are plotted in Fig. 3, where solid (dashed) lines show $R_{\mathrm{nl}}$ for a system with (without) disorder. Each curve represents a different calculation of $R_{\mathrm{nl}}$, that is, a different choice of current paths and probes used to calculate $R_{i j, k l}$. The quantized values obtained at the plateaus precisely correspond to those expected in the QSH state for the chosen injection and detection contacts, as explained in Ref. [49]. That different choices of electrical contacts yield distinct-yet precisely defined-plateau values stems from the equilibration of the chemical potential at the leads [60]; therefore, the chosen voltage probes and the current path uniquely determine the value of $R_{i j, k l}$. Note, however, that such a nonlocal setup is unable to discern the $y$ and $z$ projections of the spin in the edge states; for that, one may need to use magnetic electrodes.

We also computed the bond-projected spin currents [58] for spins polarized along the (rotated) $z^{\prime}$ and $y^{\prime}$ directions, i.e., $\boldsymbol{J}_{s}^{z^{\prime}}$ and $\boldsymbol{J}_{s}^{y^{\prime}}$. The former is shown in the inset of Fig. 3 as horizontal arrows at the top and bottom edges, evidencing the fingerprint of helical transport in the QSH regime. In contrast, $\boldsymbol{J}_{s}^{y^{\prime}}$ was found to be negligible, which is consistent with the form of $\mathcal{H}_{\mathrm{soc}}^{\prime}$ in Eq. (4). Finally, we also observe a strong resilience of the plateaus to nonmagnetic disorder (Fig. 3), consistent with time-reversal topologically protected states $(U=2 \mathrm{eV}$, much larger than any other energy scale of the Hamiltonian). These nonlocal results clearly establish that the canted QSH effect, inferred above from a bulk Kubo calculation, is characterized by robust helical spin transport at the edges, a fact fully consistent with the bulk-boundary correspondence [64].

Conclusion.-Our model building and quantum transport calculations allowed an in-depth study of the nature of spin transport in monolayers of $\mathrm{WTe}_{2}$, with emphasis on the QSH regime. Calculations of spin Hall conductivities and nonlocal resistances in multiprobe configurations revealed a so-far-unique QSH effect defined by a canted spin quantization axis, fixed by SOC characteristics. The oblique spin polarization of topological edge states in the QSH regime continuously evolves into a persistent spin texture when $E_{F}$ enters the conduction band. Our findings call for a careful analysis of QSH effect measurements, whose interpretation usually ignores the possibility of multiple nonzero components of the spin Hall conductivity tensor-as a result, noninteger quantization might be erroneously inferred by improper measurement design. A combination of measurements with applied magnetic field along different directions, or nonlocal measurements with magnetic contacts, could disentangle the different contributions of such persistent topological spin dynamics. Interestingly, such noninteger QSH plateaus were theoretically discussed for square and hexagonal lattices [62], 
suggesting the possible existence of a canted QSH effect in those systems as well.

The low-symmetry phases of TMDs may thus provide fascinating avenues to design new topological nanodevices for spin transport beyond the current paradigm of QSH effect with $z$-polarized spins. In the context of spin-orbit torque, such canted spin polarization allows for torques with different symmetries, which could facilitate the current quest for magnetic-field-free switching of magnets with out-of-plane magnetic anisotropy, a feature that is forbidden using high-symmetry 2D materials. Finally, a QSH effect whose canting angle depends on the ratio of SOC parameters could enable electrically tunable dissipationless spin currents with controllable spin orientation in the absence of magnetic fields.

M. V. acknowledges support from "La Caixa" Foundation and the Centre for Advanced 2D Materials at the National University of Singapore for its hospitality. $\mathrm{X}$.W. acknowledges the ANR Flagera GRANSPORT funding. ICN2 authors were supported by the European Union Horizon 2020 research and innovation programme under Grant Agreement No. 881603 (Graphene Flagship) and No. 824140 (TOCHA, H2020-FETPROACT-012018). ICN2 is funded by the CERCA Programme/ Generalitat de Catalunya, and is supported by the Severo Ochoa program from Spanish MINECO (Grant No. SEV2017-0706). V. M. P. acknowledges the support of the National Research Foundation (Singapore) under its Medium-Sized Centre Programme (R-723-000-001-281).

Note added.-Two experiments probing the spin polarization of $\mathrm{WTe}_{2}$ monolayers were released after submission of this manuscript $[65,66]$. They confirm that helical modes have canted spins with angles $\sim 50^{\circ}$ and $59^{\circ}$, respectively, in agreement with the value of $56^{\circ}$ predicted here.

[1] C. L. Kane and E. J. Mele, Phys. Rev. Lett. 95, 226801 (2005).

[2] C. L. Kane and E. J. Mele, Phys. Rev. Lett. 95, 146802 (2005).

[3] L. Sheng, D. N. Sheng, C. S. Ting, and F. D. M. Haldane, Phys. Rev. Lett. 95, 136602 (2005).

[4] D. N. Sheng, Z. Y. Weng, L. Sheng, and F. D. M. Haldane, Phys. Rev. Lett. 97, 036808 (2006).

[5] L. Fu, C. L. Kane, and E. J. Mele, Phys. Rev. Lett. 98, 106803 (2007).

[6] B. A. Bernevig, T. L. Hughes, and S.-C. Zhang, Science 314, 1757 (2006).

[7] B. A. Bernevig, J. Orenstein, and S.-C. Zhang, Phys. Rev. Lett. 97, 236601 (2006).

[8] M. Z. Hasan and C. L. Kane, Rev. Mod. Phys. 82, 3045 (2010).

[9] J. Sinova, S. O. Valenzuela, J. Wunderlich, C. H. Back, and T. Jungwirth, Rev. Mod. Phys. 87, 1213 (2015).

[10] Y. Fan and K. L. Wang, Spin 6, 16400014 (2016).
[11] M. König, S. Wiedmann, C. Brüne, A. Roth, H. Buhmann, L. W. Molenkamp, X.-L. Qi, and S.-C. Zhang, Science 318, 766 (2007).

[12] D. Hsieh, D. Qian, L. Wray, Y. Xia, Y. S. Hor, R. J. Cava, and M. Z. Hasan, Nature (London) 452, 970 (2008).

[13] H. Jiang, S. Cheng, Q.-f. Sun, and X. C. Xie, Phys. Rev. Lett. 103, 036803 (2009).

[14] H. Zhang, C.-X. Liu, X.-L. Qi, X. Dai, Z. Fang, and S.-C. Zhang, Nat. Phys. 5, 438 (2009).

[15] Y. Ren, Z. Qiao, and Q. Niu, Rep. Prog. Phys. 79, 066501 (2016).

[16] M. Ezawa, Phys. Lett. A 378, 1180 (2014).

[17] Ö. F. Dayi and E. Yunt, Int. J. Geom. Methods Mod. Phys. 13, 1550136 (2016).

[18] M. Konig, S. Wiedmann, C. Brune, A. Roth, H. Buhmann, L. W. Molenkamp, X.-L. Qi, and S.-C. Zhang, Science 318, 766 (2007).

[19] F. Ortmann, S. Roche, and S. Valenzuela, Topological Insulators: Fundamentals and Perspectives (Wiley-VCH, New York, 2015).

[20] S. Tang et al., Nat. Phys. 13, 683 (2017).

[21] Z. Fei, T. Palomaki, S. Wu, W. Zhao, X. Cai, B. Sun, P. Nguyen, J. Finney, X. Xu, and D. H. Cobden, Nat. Phys. 13, 677 (2017).

[22] Z.-Y. Jia, Y.-H. Song, X.-B. Li, K. Ran, P. Lu, H.-J. Zheng, X.-Y. Zhu, Z.-Q. Shi, J. Sun, J. Wen, D. Xing, and S.-C. Li, Phys. Rev. B 96, 041108(R) (2017).

[23] P. Chen, W. W. Pai, Y.-H. Chan, W.-L. Sun, C.-Z. Xu, D.-S. Lin, M. Y. Chou, A.-V. Fedorov, and T.-C. Chiang, Nat. Commun. 9, 1 (2018).

[24] S. Wu, V. Fatemi, Q. D. Gibson, K. Watanabe, T. Taniguchi, R. J. Cava, and P. Jarillo-Herrero, Science 359, 76 (2018).

[25] Y. Shi, J. Kahn, B. Niu, Z. Fei, B. Sun, X. Cai, B. A. Francisco, D. Wu, Z.-X. Shen, X. Xu, D. H. Cobden, and Y.-T. Cui, Sci. Adv. 5, eaat8799 (2019).

[26] F. Reis, G. Li, L. Dudy, M. Bauernfeind, S. Glass, W. Hanke, R. Thomale, J. Schäfer, and R. Claessen, Science 357, 287 (2017).

[27] B. Zhao, D. Khokhriakov, Y. Zhang, H. Fu, B. Karpiak, A. M. Hoque, X. Xu, Y. Jiang, B. Yan, and S. P. Dash, Phys. Rev. Research 2, 013286 (2020).

[28] C. Zhao, M. Hu, J. Qin, B. Xia, C. Liu, S. Wang, D. D. Guan, Y. Li, H. Zheng, J. Liu, and J. Jia, Phys. Rev. Lett. 125, 046801 (2020).

[29] D. Khokhriakov, A. M. Hoque, B. Karpiak, and S. P. Dash, Nat. Commun. 11, 3657 (2020).

[30] A. M. Hoque, D. Khokhriakov, B. Karpiak, and S. P. Dash, Phys. Rev. Research 2, 033204 (2020).

[31] L. Li, J. Zhang, G. Myeong, W. Shin, H. Lim, B. Kim, S. Kim, T. Jin, S. Cavill, B. S. Kim, C. Kim, J. Lischner, A. Ferreira, and S. Cho, ACS Nano 14, 5251 (2020).

[32] X. Qian, J. Liu, L. Fu, and J. Li, Science 346, 1344 (2014).

[33] S. Singh, J. Kim, K. M. Rabe, and D. Vanderbilt, Phys. Rev. Lett. 125, 046402 (2020).

[34] Y. Zhang, J. van den Brink, C. Felser, and B. Yan, 2D Mater. 5, 044001 (2018).

[35] B. Zhao, B. Karpiak, D. Khokhriakov, A. Johansson, A. M. Hoque, X. Xu, Y. Jiang, I. Mertig, and S. P. Dash, Adv. Mater. 32, 2000818 (2020). 
[36] K. v. Klitzing, G. Dorda, and M. Pepper, Phys. Rev. Lett. 45, 494 (1980).

[37] L. Peng, Y. Yuan, G. Li, X. Yang, J.-J. Xian, C.-J. Yi, Y.-G. Shi, and Y.-S. Fu, Nat. Commun. 8, 659 (2017).

[38] S. Ok, L. Muechler, D. Di Sante, G. Sangiovanni, R. Thomale, and T. Neupert, Phys. Rev. B 99, 121105(R) (2019).

[39] J. I. Väyrynen, D. I. Pikulin, and J. Alicea, Phys. Rev. Lett. 121, 106601 (2018).

[40] P. Novelli, F. Taddei, A. K. Geim, and M. Polini, Phys. Rev. Lett. 122, 016601 (2019).

[41] L. Vannucci, T. Olsen, and K. S. Thygesen, Phys. Rev. B 101, 155404 (2020).

[42] J. E. Hirsch, Phys. Rev. Lett. 83, 1834 (1999).

[43] C. K. Safeer, N. Ontoso, J. Ingla-Aynés, F. Herling, V. T. Pham, A. Kurzmann, K. Ensslin, A. Chuvilin, I. Robredo, M. G. Vergniory, F. de Juan, L. E. Hueso, M. R. Calvo, and F. Casanova, Nano Lett. 19, 8758 (2019).

[44] P. Song, C.-H. Hsu, G. Vignale, M. Zhao, J. Liu, Y. Deng, W. Fu, Y. Liu, Y. Zhang, H. Lin, V. M. Pereira, and K. P. Loh, Nat. Mater. 19, 292 (2020).

[45] M. Seemann, D. Ködderitzsch, S. Wimmer, and H. Ebert, Phys. Rev. B 92, 155138 (2015).

[46] T. P. Cysne, A. Ferreira, and T. G. Rappoport, Phys. Rev. B 98, 045407 (2018).

[47] J. Schliemann, J. C. Egues, and D. Loss, Phys. Rev. Lett. 90, 146801 (2003).

[48] J. Schliemann, Rev. Mod. Phys. 89, 011001 (2017).

[49] See Supplemental Material at http://link.aps.org/ supplemental/10.1103/PhysRevLett.125.256603 for details of the model and a discussion on the symmetries.

[50] S.-Y. Xu, Q. Ma, H. Shen, V. Fatemi, S. Wu, T.-R. Chang, G. Chang, A. M. M. Valdivia, C.-K. Chan, Q. D. Gibson, J. Zhou, Z. Liu, K. Watanabe, T. Taniguchi, H. Lin, R. J. Cava, L. Fu, N. Gedik, and P. Jarillo-Herrero, Nat. Phys. 14, 900 (2018).

[51] L.-K. Shi and J. C. W. Song, Phys. Rev. B 99, 035403 (2019).
[52] J. Heyd, G. E. Scuseria, and M. Ernzerhof, J. Chem. Phys. 118, 8207 (2003).

[53] J. H. Garcia, M. Vila, A. W. Cummings, and S. Roche, Chem. Soc. Rev. 47, 3359 (2018).

[54] A. Bastin, C. Lewiner, O. Betbeder-matibet, and P. Nozieres, J. Phys. Chem. Solid 32, 1811 (1971).

[55] A. Cresti, B. K. Nikolić, J. H. García, and S. Roche, Riv. Nuovo Cimento 39, 587 (2016).

[56] Z. Fan, J. Garcia, A. Cummings, J. Barrios-Vargas, M. Panhans, A. Harju, F. Ortmann, and S. Roche, arXiv:1811 .07387 .

[57] J. H. García, L. Covaci, and T. G. Rappoport, Phys. Rev. Lett. 114, 116602 (2015).

[58] C. W. Groth, M. Wimmer, A. R. Akhmerov, and X. Waintal, New J. Phys. 16, 063065 (2014).

[59] M. Vila, J. H. Garcia, A. W. Cummings, S. R. Power, C. W. Groth, X. Waintal, and S. Roche, Phys. Rev. Lett. 124, 196602 (2020).

[60] A. Roth, C. Brüne, H. Buhmann, L. W. Molenkamp, J. Maciejko, X.-L. Qi, and S.-C. Zhang, Science 325, 294 (2009).

[61] L. Matthes, S. Küfner, J. Furthmüller, and F. Bechstedt, Phys. Rev. B 94, 085410 (2016).

[62] F. Matusalem, M. Marques, L. K. Teles, L. Matthes, J. Furthmüller, and F. Bechstedt, Phys. Rev. B 100, 245430 (2019).

[63] S. Datta, Electronic Transport in Mesoscopic Systems (Cambridge University Press, Cambridge, England, 1997).

[64] Y. Hatsugai, Phys. Rev. Lett. 71, 3697 (1993).

[65] W. Zhao, E. Runburg, Z. Fei, J. Mutch, P. Malinowski, B. Sun, X. Huang, D. Pesin, Y.-T. Cui, X. Xu, J.-H. Chu, and D. H. Cobden, arXiv:2010.09986

[66] C. Tan, M.-X. Deng, F. Xiang, G. Zheng, S. Albarakati, M. Algarni, J. Partridge, A. R. Hamilton, R.-Q. Wang, and L. Wang, arXiv:2010.15717 